\title{
A versatile photodetector assisted by photovoltaic and bolometric effects
}

\author{
Wei Jiang $\mathbb{1}^{1,2}$, Tan Zheng ${ }^{3}$, Binmin $\mathrm{Wu}^{1}$, Hanxue Jiao ${ }^{1}$, Xudong Wang ${ }^{1}$, Yan Chen ${ }^{1}$, Xiaoyu Zhang ${ }^{1}$, Meng Peng ${ }^{1}$, \\ Hailu Wang ${ }^{1,2}$, Tie Lin', Hong Shen', Jun Ge', Weida Hu ${ }^{1}{ }^{1}$, Xiaofeng X $\mathrm{u}^{3}$, Xiangjian Meng ${ }^{1}$, Junhao Chu ${ }^{1}$ and \\ Jianlu Wang (10 ${ }^{1}$
}

\begin{abstract}
The advent of low-dimensional materials with peculiar structure and superb band properties provides a new canonical form for the development of photodetectors. However, the limited exploitation of basic properties makes it difficult for devices to stand out. Here, we demonstrate a hybrid heterostructure with ultrathin vanadium dioxide film and molybdenum ditelluride nanoflake. Vanadium dioxide is a classical semiconductor with a narrow bandgap, a high temperature coefficient of resistance, and phase transformation. Molybdenum ditelluride, a typical two-dimensional material, is often used to construct optoelectronic devices. The heterostructure can realize three different functional modes: (i) the p-n junction exhibits ultrasensitive detection ( $450 \mathrm{~nm}-2 \mu \mathrm{m})$ with a dark current down to $0.2 \mathrm{pA}$ and a response time of $17 \mu \mathrm{s}$, (ii) the Schottky junction works stably under extreme conditions such as a high temperature of $400 \mathrm{~K}$, and (iii) the bolometer shows ultrabroad spectrum detection exceeding $10 \mu \mathrm{m}$. The flexible switching between the three modes makes the heterostructure a potential candidate for next-generation photodetectors from visible to longwave infrared radiation (LWIR). This type of photodetector combines versatile detection modes, shedding light on the hybrid application of novel and traditional materials, and is a prototype of advanced optoelectronic devices.
\end{abstract}

\section{Introduction}

Photodetectors ${ }^{1}$, due to their widespread application and superior status, have emerged as a research hotspot since their appearance in the early 1910s. According to the energy conversion process, photodetectors can generally be categorized as quantum detectors and thermal detectors $^{1-4}$. Quantum detectors can directly convert photons into electric signals, while thermal detectors generate electric output through changes in physical properties under incident radiation. Depending on the detection mechanism, the class of photodetectors can be further divided into different types, including photoconductive detectors ( $\mathrm{PbSe}, \mathrm{PbS}, \mathrm{HgCdTe}, \mathrm{InSb}$ ),

\footnotetext{
Correspondence: Weida Hu (wdhu@mail.sitp.ac.cn) or Xiaofeng Xu (xxf@dhu.edu.cn) or Jianlu Wang (jlwang@mail.sitp.ac.cn)

${ }^{1}$ State Key Laboratory of Infrared Physics, Shanghai Institute of Technical Physics, Chinese Academy of Sciences, 500 Yutian Road, Shanghai 200083, China

${ }^{2}$ University of Chinese Academy of Sciences, Beijing 100049, China Full list of author information is available at the end of the article
}

photovoltaic detectors ( $\mathrm{Si}, \mathrm{Ge}, \mathrm{HgCdTe}$, InAs, InSb), and quantum well detectors (GaAs/AlGaAs). The thermal detectors also include thermopiles (Pt-Ir), bolometers $\left(\mathrm{VO}_{2}, \alpha-\mathrm{Si}\right)$, and pyroelectric detectors $\left(\mathrm{LiTaO}_{3}\right.$, PVDF) ${ }^{2,3,5,6}$. Recently, the emergence of detectors based on low-dimensional materials ${ }^{7-12}$, such as quantum dots, nanowires, nanoflakes, and nanofilms, has led to a boom in the development of excellent photodetectors. Nanostructured materials with reduced physical size and unique band structure bring about incredible performance compared to traditional materials. In particular, twodimensional (2D) semiconductors have received more attention for their plane characteristics and lack of dangling bonds ${ }^{13}$, which is an apparent advantage for building various vertical heterostructures ${ }^{14}$ and integration with $\mathrm{CMOS}^{15}$. In fact, promising progress has been made in the development of $2 \mathrm{D}$ photodetectors ${ }^{16,17}$. Photodetectors based on graphene ${ }^{18}$, transition metal chalcogenides $(\mathrm{TMDCs})^{19}$, and black phosphorus ${ }^{20}$ exhibit

\section{(c) The Author(s) 2020}

(c) (i) Open Access This article is licensed under a Creative Commons Attribution 4.0 International License, which permits use, sharing, adaptation, distribution and reproduction in any medium or format, as long as you give appropriate credit to the original author(s) and the source, provide a link to the Creative Commons license, and indicate if changes were made. The images or other third party material in this article are included in the article's Creative Commons license, unless indicated otherwise in a credit line to the material. If material is not included in the article's Creative Commons license and your intended use is not permitted by statutory regulation or exceeds the permitted use, you will need to obtain permission directly from the copyright holder. To view a copy of this license, visit http://creativecommons.org/licenses/by/4.0/. 
ultrafast responses, exceptional sensitivities, or broadspectrum responses. Localized field modulation ${ }^{21,22}$ and heterojunction construction ${ }^{23,24}$ are usually applied to enhance various detector performances. However, there is no evidence of a detector with excellent comprehensive performance. According to the demands for nextgeneration infrared detectors ${ }^{2}$, can we develop an uncooled versatile detector with rapid response, high sensitivity, and broadband response?

Here, a new concept of a photodetector combining photovoltaic detectors with a bolometer is demonstrated. A photon with energy larger than the bandgap can be directly absorbed by an electron in the valence band. Then, the electron undergoes an optical transition to the conductance band. An electron-hole pair will be generated after the transition and separated by a built-in electric field or a bias voltage, which is the origin of the photocurrent of the photovoltaic detector. Due to the rapid separation of electron-hole pairs, the photovoltaic detector is known for its sensitivity, despite its limited response range. When the energy of mid-infrared or farinfrared radiation is not sufficient to allow the electron to undergo an optical transition, electron-phonon interactions occur between phonons and low-energy photons. Therefore, the thermal equilibrium and carrier density are disturbed, causing fluctuations in resistance. This is the so-called bolometric effect, which is particularly significant in materials with a high temperature coefficient of resistance, such as $\mathrm{VO}_{2}{ }^{25}$. Compared to the photovoltaic detector, the bolometer is not wavelength selective, but the response rate is relatively slow. To combine the advantages of the two detector types, a heterostructure device based on $\mathrm{VO}_{2}$ and $\mathrm{MoTe}_{2}$ is fabricated. There are three operation modes integrated into one detector, including the $\mathrm{p}-\mathrm{n}$ junction mode, Schottky junction mode, and bolometer mode. The three modes can be flexibly switched between for different situations. This detector achieves detection from visible light to longwave infrared radiation (LWIR) and simultaneously exhibits good performance in terms of a rapid response and a high sensitivity.

\section{Results}

\section{Device structure and characterization}

$\mathrm{VO}_{2}{ }^{25-27}$ is a classic phase transition material with a metal-insulator transition (MIT) near room temperature $(340 \mathrm{~K})$. When the temperature is below the transformation temperature $\left(T_{c}\right), \mathrm{VO}_{2}$ is in a monoclinic phase $\mathrm{e}^{28}$. The $\mathrm{O}$ atoms are in different chemical states, as labeled in Fig. 1a. In this phase, the Fermi level is in the middle of the $d_{\|}$bonding orbital and $d_{\|}{ }^{*}$ antibonding orbital ${ }^{29}$, indicating that $\mathrm{VO}_{2}$ is a semiconductor. When the a

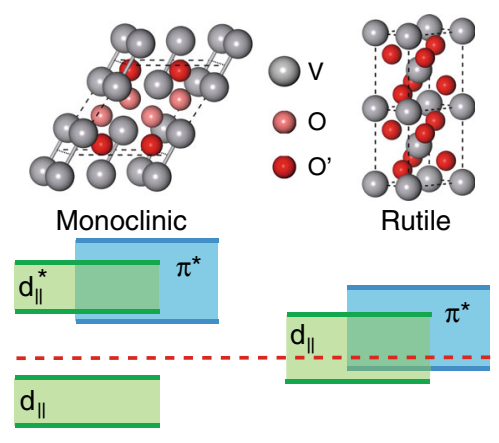

C

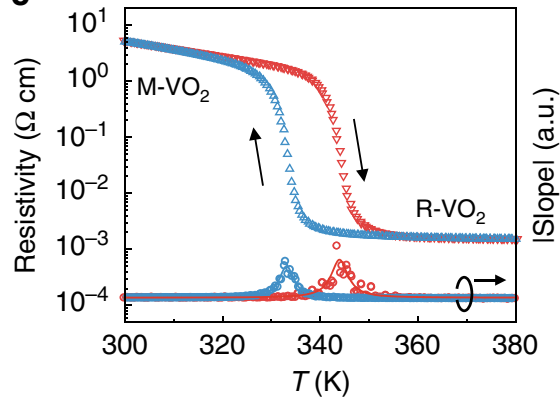

b

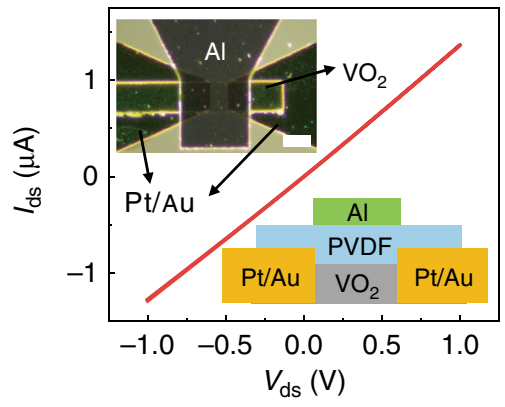

d

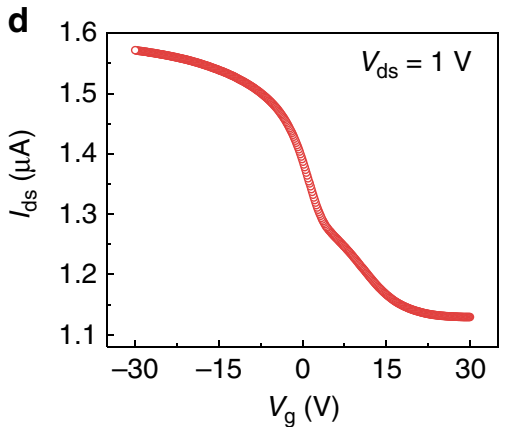

Fig. 1 Structure and electronic characterization of $\mathbf{V O}_{2}$. a Schematic of the crystal structure and band structure of monoclinic and rutile $\mathrm{VO}_{2}$. $\mathbf{b}$ Output characteristics of the P(VDF-TrFE)-gated $\mathrm{VO}_{2}$ FET; the insets show the schematic and corresponding optical image. Scale bar, $20 \mu \mathrm{m}$. $\mathbf{c}$ Resistivity of the $\mathrm{VO}_{2}$ film measured under vacuum as a function of temperature (red, heating; blue, cooling). The lower points and fitted curves show the abs(slope) of the resistivity. $\mathbf{d}$ Transfer characteristics at $V_{\mathrm{ds}}=1 \mathrm{~V}$ of the $\mathrm{VO}_{2} \mathrm{FET}$. 


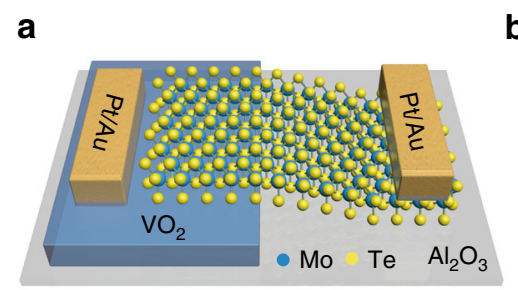

b

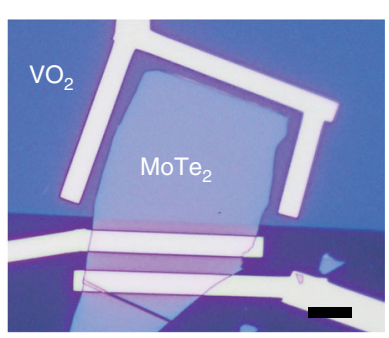

C

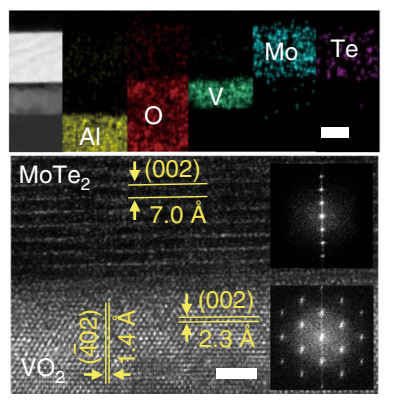

d

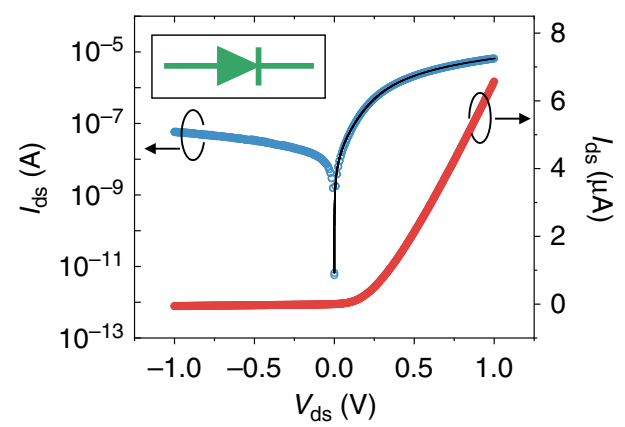

Fig. 2 Structure and properties of the heterostructure. $\mathbf{a}$, $\mathbf{b}$ Schematic and optical micrograph of the $\mathrm{VO}_{2} / \mathrm{MoTe}_{2}$ heterostructure on a sapphire substrate. Scale bar, $10 \mu \mathrm{m}$. c Cross-sectional TEM image and corresponding EDS mappings of the heterostructure. Scale bar, $20 \mathrm{~nm}$ (top); an HRTEM image of the interface of the heterostructure is shown at the bottom. The insets show the corresponding fast Fourier transform patterns indexed to $\mathrm{MoTe}_{2}$ and $\mathrm{VO}_{2}$. Scale bar, $2 \mathrm{~nm}$ (bottom). $\mathbf{d}$ Linear plots (red) and logarithmic plots (blue) of $I_{\mathrm{ds}}$ as a function of $V_{\mathrm{ds}}$. The inset symbol represents the junction mode.

temperature is above $T_{c}, \mathrm{VO}_{2}$ transforms to the rutile phase, and the $\mathrm{O}$ atoms are in the same chemical state. In this phase, the Fermi level passes through the overlap zone of the $d_{\|}$orbital and $\pi$ " orbital. As a result, $\mathrm{VO}_{2}$ shows metallic behavior.

Here, the $\mathrm{VO}_{2}$ film is synthesized on a sapphire substrate (see "Materials and methods"). The thickness is measured to be $\sim 22 \mathrm{~nm}$ with a surface undulation of $<2 \mathrm{~nm}$ (Fig. S1). As shown in Fig. S2a, X-ray diffraction (XRD) and Raman spectroscopy are performed to identify the phase and purity. The peaks marked (002) and (402) in Fig. S2a result from the monoclinic phase $\mathrm{VO}_{2}{ }^{30}$. Raman peaks are distinguished in Fig. S2b, but all of them can be ascribed to the vibration modes of $\mathrm{VO}_{2}{ }^{31}$, indicating that the sample is a high-quality $\mathrm{VO}_{2}$ film with no other vanadium oxides. First, the basic electrical properties of $\mathrm{VO}_{2}$ are investigated by using a top-gated fieldeffect transistor (FET) with poly(vinylidene fluoride-trifluoroethylene) (P(VDF-TrFE)) as a dielectric layer, as shown in Fig. 1b. The output curve in Fig. 1b indicates that the $\mathrm{Pt} / \mathrm{Au}$ electrode can form good ohmic contact with $\mathrm{VO}_{2}$. As shown in Fig. 1c, the resistivity changes by more than $10^{3}$ between monoclinic $\mathrm{VO}_{2}\left(\mathrm{M}-\mathrm{VO}_{2}\right)$ and rutile $\mathrm{VO}_{2}\left(\mathrm{R}-\mathrm{VO}_{2}\right)$, showing a significant relationship with temperature. A hysteresis phenomenon appears during thermal cycling. The phase transition temperature is $\sim 344 \mathrm{~K}$ during the heating process and $\sim 333 \mathrm{~K}$ during the cooling process, extracted from the slope of the temperature-dependent resistivity curve. The temperature of the semiconductor to metal transformation is slightly higher than $340 \mathrm{~K}$ because of the $\operatorname{strain}^{32}$ between domains as well as the inhomogeneous strain fields with the substrate ${ }^{33}$. Figure $1 \mathrm{~d}$ shows the transfer characteristic curve. The $\mathrm{VO}_{2}$ film behaves as an unusual p-type material, but this behavior can be achieved by adjusting the temperature in the synthesis process ${ }^{34}$.

Figure 2a shows a schematic of our heterostructure. A thick $\mathrm{MoTe}_{2}$ flake is chosen to fabricate a heterostructure with $\mathrm{VO}_{2}$ because it always behaves as an $\mathrm{n}$-type material $^{35}$ and its bandgap is relatively narrow compared to other TMDCs ${ }^{16}$. Figure $2 \mathrm{~b}$ shows an optical image of the fabricated heterostructure. The thickness of $\mathrm{MoTe}_{2}$ is identified by AFM to be $\sim 40 \mathrm{~nm}$ (Fig. S3a). Figure S3b presents the Raman modes of the typical thick $\mathrm{MoTe}_{2}$, in which the intensity of the $A_{1 g}$ vibration is much weaker than that of the $E_{2 g}$ vibration mode ${ }^{36}$. Finally, P(VDFTrFE) is spin-coated on the device to protect it from oxygen. With such optimization, a heterostructure between $\mathrm{VO}_{2}$ and $\mathrm{MoTe}_{2}$ is achieved. A cross-sectional TEM image and the corresponding energy dispersive $\mathrm{X}$ ray spectroscopy (EDS) mappings of the $\mathrm{MoTe}_{2} / \mathrm{VO}_{2}$ heterostructure are shown at the top of Fig. 2c. Each component is identified and marked in the images. From left to right, they are $\mathrm{Al}, \mathrm{O}, \mathrm{V}, \mathrm{Mo}$, and Te. This 
heterostructure is a tightly stacked structure with almost no impurity layer observed. The detailed atomic structures of $\mathrm{VO}_{2}$ and $\mathrm{MoTe}_{2}$ are investigated by highresolution TEM, as shown at the bottom of Fig. 2c. The two insets are fast Fourier transform patterns of the $\mathrm{VO}_{2}$ and $\mathrm{MoTe}_{2}$ data. $\mathrm{MoTe}_{2}$ has a clear layered structure, and the layer-to-layer spacing is $\sim 0.70 \mathrm{~nm}^{37}$. $\mathrm{VO}_{2}$ also shows high crystallinity, and the $d$-spacings of (002) and (402) are 0.23 and $0.14 \mathrm{~nm}^{38}$, respectively, corresponding to the XRD pattern.

Figure $2 \mathrm{~d}$ shows the output characteristics of this device. The classical diode output curve (red) proves the existence of a junction. A rectification factor of $10^{2}$ can be achieved, shown on a logarithmic scale (blue curve). The curve is fitted by the following $\mathrm{p}-\mathrm{n}$ junction diode equation including a series resistance $R_{s}^{39}$.

$$
I_{d s}=\frac{n V_{T}}{R_{s}} W\left[\frac{I_{0} R_{s}}{n V_{T}} \exp \left(\frac{V_{d s}+I_{0} R_{s}}{n V_{T}}\right)\right]-I_{0}
$$

in which $V_{T}=k_{b} T / e$ is the thermal voltage at temperature $\mathrm{K}, k_{\mathrm{B}}$ is the Boltzmann constant, $e$ is the electron charge, $I_{0}$ is the reverse bias current, $n$ is the ideality factor, and $W$ is the Lambert $W$ function. In this heterostructure, $n$ is fitted as 2.2 (for an ideal p-n junction, $n=1$ ) with an $R_{s}$ of $0.99 \mathrm{M} \Omega$, indicating that the transport characteristics are dominated by recombination, probably because of the large density of surface trap states from the polycrystalline $\mathrm{VO}_{2}$ film acting as recombination centers ${ }^{40}$. To further prove that a junction is generated between $\mathrm{VO}_{2}$ and $\mathrm{MoTe}_{2}$, the contact and electrical transport properties of fractional $\mathrm{VO}_{2}$ and $\mathrm{MoTe}_{2}$ are measured and presented in Fig. S4. As shown in Fig. S4a, b, ohmic contact is simultaneously achieved for $\mathrm{VO}_{2}$ and $\mathrm{MoTe}_{2}$, which means that the junction behavior only results from the $\mathrm{VO}_{2} / \mathrm{MoTe}_{2}$ heterojunction part. Furthermore, to prove that it is a $\mathrm{p}-\mathrm{n}$ junction, the transfer characteristics of $\mathrm{MoTe}_{2}$ are measured in a $\mathrm{P}(\mathrm{VDF}-\mathrm{TrFE})$-gated FET. Graphene is transferred as a top electrode (Fig. S4c). P (VDF-TrFE) is not only a protective membrane but also a powerful dielectric layer with a strong polarization electric field ${ }^{41}$. As shown in Fig. S4d, when the gate voltage $\left(V_{g}\right)$ is switched from -30 to $30 \mathrm{~V}$, the current between the drain and source $\left(I_{\mathrm{ds}}\right)$ increases logarithmically, conforming to the n-type behavior of thick $\mathrm{MoTe}_{2}$. As $\mathrm{VO}_{2}$ is p-type and thick $\mathrm{MoTe}_{2}$ has n-type characteristics, the heterostructure is a typical $\mathrm{p}-\mathrm{n}$ junction with a built-in electric field directed from $\mathrm{MoTe}_{2}$ to $\mathrm{VO}_{2}$.

\section{Photoresponse in the $p-n$ junction mode}

Figure 3a shows the output characteristics under $830 \mathrm{~nm}$ illumination of various laser powers. The open-
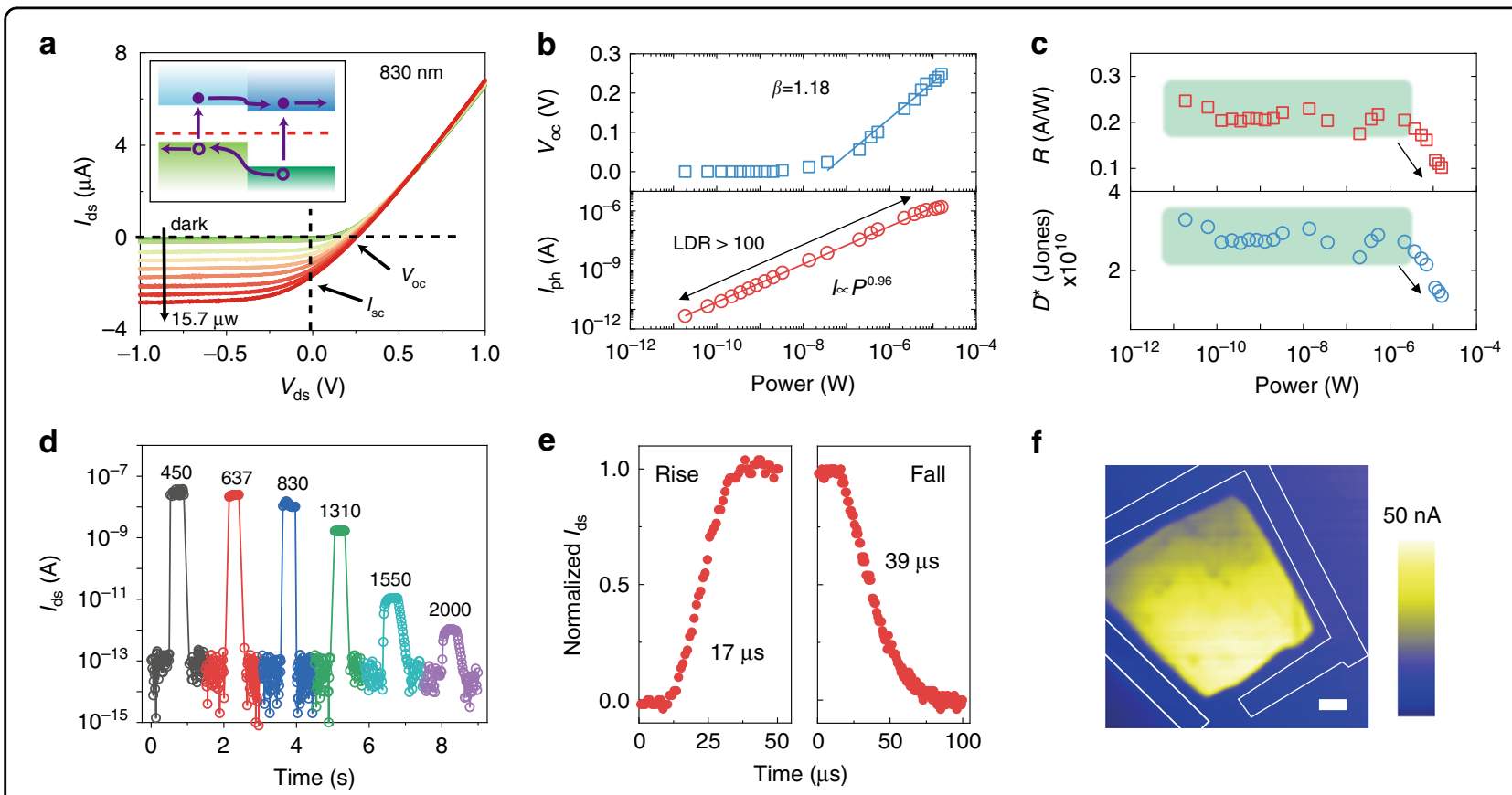

Fig. 3 Power and wavelength dependence of the $\mathbf{p}-\mathbf{n}$ junction mode. $\mathbf{a} I_{\mathrm{ds}}-V_{\mathrm{ds}}$ curves for incident laser powers from 0 to $15.7 \mu \mathrm{W}$. The inset shows a schematic of the photovoltaic mechanism for detecting visible to near-infrared radiation. $\mathbf{b} V_{\mathrm{OC}}$ and $I_{\mathrm{ph}}$ as a function of the incident laser power. c Responsivity and detectivity as a function of the incident laser power. $\mathbf{d}$ Temporal response of the photocurrent under laser illumination of $450,637,830,1310,1550$, and $2000 \mathrm{~nm}$; all the powers are fixed at $80 \mathrm{nW}$. e Rise and fall times of the normalized photocurrent under zero bias measured by an oscilloscope. $\mathbf{f}$ Mapping of the photocurrent measured under scanning laser illumination at $830 \mathrm{~nm}$; the yellow area corresponds to the overlap of the heterostructure. Scale bar, $5 \mu \mathrm{m}$. 
circuit voltage $\left(V_{\mathrm{oc}}\right)$ and short-circuit current $\left(I_{\mathrm{sc}}\right)$ increase as the power increases from 0 to $15.7 \mu \mathrm{W}$. Due to the photovoltaic effect, the electron-hole pairs generated by electron transition are separated by the built-in electric field and collected by the electrodes (inset of Fig. 3a). Herein, $\left|I_{\mathrm{ds}}\right|$ increases to $\sim 2 \mu \mathrm{A}$ at zero bias under 15.7$\mu \mathrm{W}$ illumination, which is several orders of magnitude higher than the dark current of $2 \mathrm{pA}$. To investigate the power dependence of our device, $V_{\mathrm{oc}}$ and $I_{\mathrm{ph}}\left(I_{\mathrm{ph}}=I_{\text {dark }}\right.$ $\left.-I_{\mathrm{sc}}\right)$ for various powers are plotted and fitted in Fig. $3 \mathrm{~b}$. $V_{\text {oc }}$ increases with power, excluding the small values outside of the experimental voltage resolution. $V_{\mathrm{oc}}$ can be described by the following equation ${ }^{42}$ :

$$
\frac{d V_{\mathrm{oc}}}{d \log (P)}=2.3 \times \frac{2}{\beta} \frac{k_{b} T}{e},
$$

where $\beta$ is the recombination order. $\beta=1$ represents Shockley-Read-Hall (SRH) recombination, and $\beta=2$ indicates Langevin recombination. The experimental data are fitted, and $\beta$ is evaluated as 1.18 , indicating that the SRH (monomolecular) process dominated recombination. In other words, a large density of the charge-trap states of $\mathrm{MoTe}_{2}$ or $\mathrm{VO}_{2}$ participated in recombination. $I_{\mathrm{ph}}$ shows a linear dependence on power, complying with the power law $I_{\mathrm{ph}}=P^{\alpha}$, where $\alpha$ is calculated to be 0.96 . This value is very close to the ideal value, 1 , indicating an efficient process of electron-hole pair generation and recombination. The linear dynamic range of this device is $>100$, exceeding that of most $2 \mathrm{D}$ material-based heterostructures ${ }^{42,43}$. The output electric power $P_{\mathrm{el}}=I_{\mathrm{ds}} V_{\mathrm{ds}}$ as a function of $V_{\mathrm{ds}}$ is plotted in Fig. S5a. The fill factor FF $=$ $P_{\mathrm{el}, \max } /\left(I_{\mathrm{sc}} V_{\mathrm{oc}}\right)\left(P_{\mathrm{el}, \max }\right.$ is the maximum power point in Fig. S5a) and the power conversion efficiency $\eta=P_{\mathrm{el}, \max } /$ $P$ are extracted and plotted in Fig. S5b, c. It is shown that FF is maintained between 0.24 and 0.32 under different incident laser powers, and the value of $\eta$ is $\sim 0.90 \%$ on average. Figure 3c shows the power-dependent responsivity $(R)$ and detectivity $\left(D^{\prime \prime}\right)^{43}$ of our heterostructure, which are defined as:

$$
\begin{aligned}
& R=\frac{I_{\mathrm{ph}}}{P}, \\
& D^{*}=\frac{R \sqrt{S}}{\sqrt{2 e I_{\text {dark }}}} .
\end{aligned}
$$

$S$ is the overlapped area of the heterostructure $\left(1133 \mu \mathrm{m}^{2}\right), e$ is the electron charge, and $I_{\text {dark }}$ is the dark current, which we evaluated as $0.2 \mathrm{pA}$. Here, $R$ is calculated to be $0.22 \mathrm{~A} / \mathrm{W}$ on average before absorbance saturation and decreases to $0.1 \mathrm{~A} / \mathrm{W}$ after saturation. $D^{*}$ shows the same tendency as $R$, and the average of $D^{*}$ is $\sim 3 \times 10^{10}$ Jones. The external quantum efficiency (EQE) is the ratio of the number of collected carriers to the number of incident photons, determined by the equation $\mathrm{EQE}=R h \mathrm{c} / \lambda e$, where $\lambda$ is the wavelength of light, $h$ is Planck's constant, and $c$ is the speed of light. The EQE at $830 \mathrm{~nm}$ is $\sim 30 \%$ before saturation (Fig. S5d), comparable to that of reported $2 \mathrm{D} p-\mathrm{n}$ junctions ${ }^{44}$.

Figure 3d exhibits the rapid temporal response at different wavelengths $(\lambda)$ with a fixed power of $80 \mathrm{nW}$. The photocurrent shows a remarkable and rapid change between dark state and laser illumination, even though the on/off ratio decreases under near-infrared radiation. However, the heterostructure still behaves excellently under $2 \mu \mathrm{m}$ laser illumination with an on/off ratio of more than 10 , which is incredible for most $2 \mathrm{D} \mathrm{p}-\mathrm{n}$ diodes ${ }^{45,46}$. The responsivity for various wavelengths is calculated and presented in Fig. S6a. The device shows a broadband response of the photovoltaic effect from 0.45 to $2 \mu \mathrm{m}$. A rapid decrease occurs when the wavelength ranges from 1.31 to $2 \mu \mathrm{m}$ because the bandgap of bulk $\mathrm{MoTe}_{2}$ is $\sim 0.9 \mathrm{eV}$ and only $\mathrm{VO}_{2}$ contributes to the responsivity when $\lambda$ is longer than $1.31 \mu \mathrm{m}$. In addition, the cutoff $\lambda$ here is $2 \mu \mathrm{m}$, very close to the bandgap of $\mathrm{VO}_{2}$, estimated from the absorbance spectrum in Fig. S6b. As the response time exceeds the limit of the photoelectric measurement system, an oscilloscope is introduced to measure the rise and fall times of the photocurrent under zero bias. As shown in Fig. 3e, the rise and fall times are evaluated as 17 and $39 \mu \mathrm{s}$, respectively, which are quicker than those of other $\mathrm{MoTe}_{2}$ - or $\mathrm{VO}_{2}$-based heterostructures ${ }^{43,47}$ as a result of rapid separation and recombination. However, the response time here is also limited by the preamplifier or oscilloscope ${ }^{48}$, and it may be faster. The response times for other wavelengths are shown in Fig. S6c. The response rate becomes slower when the wavelength exceeds $1.31 \mu \mathrm{m}$, ascribed to the influence of the bolometric effect. Scanning photocurrent microscopy is an effective method to identify the area where photocurrent is generated. $I_{\mathrm{ds}}$ at zero bias is measured as a laser spot scans the surface of the heterostructure. Figure $3 \mathrm{f}$ shows the mapping of the measured photocurrent of this $\mathrm{p}-\mathrm{n}$ junction, in which the bright yellow area represents the area where spontaneous charges are separated. It is clear that the area is the overlap of $\mathrm{MoTe}_{2}$ and $\mathrm{VO}_{2}$, further proving that the photoresponse is dominated by the photovoltaic effect from the $\mathrm{MoTe}_{2} / \mathrm{VO}_{2}$ junction. Photocurrent mappings for other wavelengths and devices are shown in Figs. S7 and S8, proving the repeatability and high performance of our devices.

\section{Bolometer mode}

The photovoltaic detector is a device that works under zero or negative bias, but here, the $\mathrm{p}-\mathrm{n}$ junction under forward bias can also be used. When the forward bias is larger than the built-in electric field, the $\mathrm{p}-\mathrm{n}$ junction can 


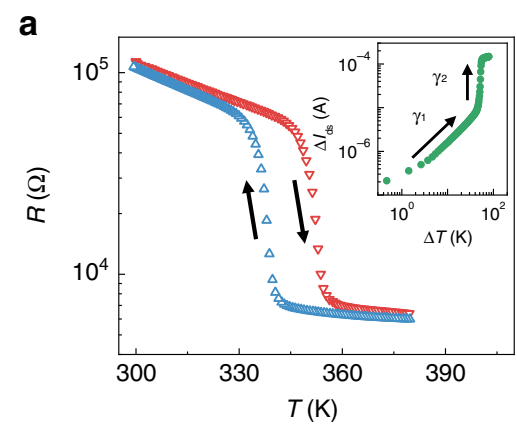

d

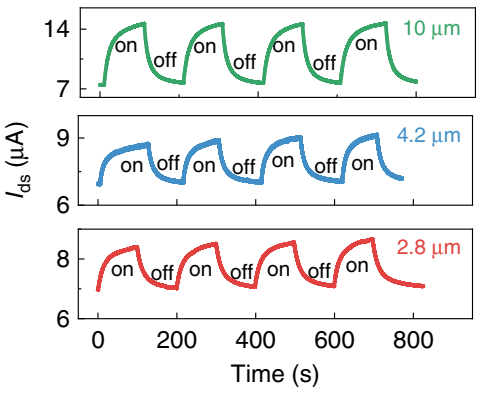

b

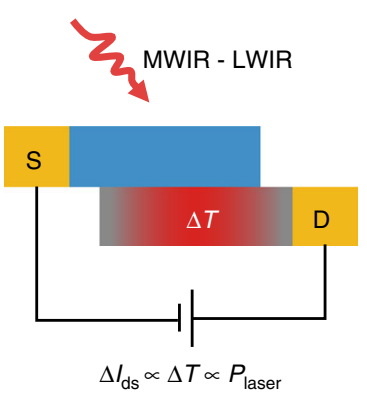

e

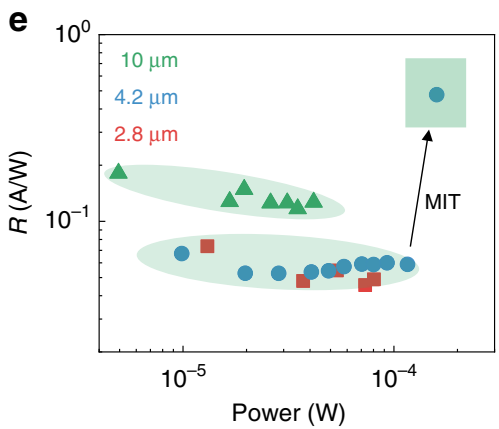

C

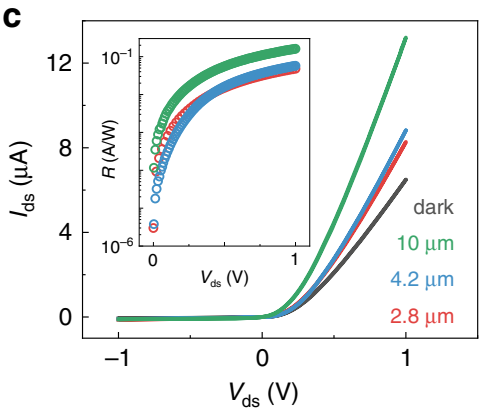

f

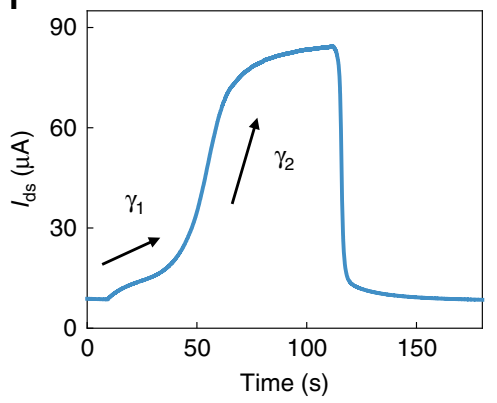

Fig. 4 Bolometer mode of the heterostructure. a Resistance of the $\mathrm{VO}_{2} / \mathrm{MoTe}_{2}$ heterostructure as a function of temperature (red, heating; blue, cooling). The inset shows the extracted $\Delta I_{\mathrm{ds}}$ and $\Delta T$, in which $\Delta I_{\mathrm{ds}}$ increases with $\Delta T$ with slopes of $\gamma_{1}$ and $\gamma_{2}$. $\mathbf{b}$ Schematic of the bolometer mode for detecting MWIR to LWIR. $\mathbf{c} I_{\text {ds }}-V_{\text {ds }}$ curves for different incident laser wavelengths (black, dark; green, $10 \mu \mathrm{m}, 41.6 \mu \mathrm{W}$; blue, $4.2 \mu \mathrm{m}$, $40.6 \mu \mathrm{W}$; red, $2.8 \mu \mathrm{m}, 37.1 \mu \mathrm{W}$ ). The inset shows the calculated responsivity for the three wavelengths as a function of $V_{\text {ds. }}$. $\mathbf{d}$ Temporal response of the photocurrent under laser illumination of $10 \mu \mathrm{m}, 4.2 \mu \mathrm{m}$, and $2.8 \mu \mathrm{m}$. e Power dependence of the heterostructure. The responsivity rapidly increases when the laser power exceeds $157 \mu \mathrm{W}$, indicating the metal-insulator transformation. f Temporal response of the photocurrent under high-power laser illumination of $157 \mu \mathrm{W}$.

be considered a resistor as the SCR of the $\mathrm{p}-\mathrm{n}$ junction disappears. The device transforms into a bolometer because of the presence of $\mathrm{VO}_{2}$. First, $I_{\mathrm{ds}}$ at $V_{\mathrm{ds}}=1 \mathrm{~V}$ during thermal cycling is measured from 300 to $380 \mathrm{~K}$ and back to $300 \mathrm{~K}$. As shown in Fig. 4a, the resistance of this heterostructure is calculated and plotted as a function of temperature. The resistance shows a rapid decrease with increasing temperature, especially in the MIT range. Therefore, our device is capable of infrared detection similar to the $\mathrm{VO}_{2}$ bolometer. As the detection of infrared radiation is a process of decreasing resistance, $\Delta I$ and $\Delta T$ are extracted and shown in the inset of Fig. 4a. It is clear that $\Delta I$ increases with a slope of $\gamma_{1}$ before the MIT and increases rapidly during the MIT with a slope of $\gamma_{2}$, in which $\gamma_{2}>\gamma_{1}$. Figure $4 \mathrm{~b}$ shows the infrared detection mechanism of our device. When under laser illumination below energy causing the MIT, the carrier density of $\mathrm{VO}_{2}$ changes after absorbing energy from mid-wavelength infrared radiation (MWIR) to LWIR. The increased carriers drift to opposite electrodes in the external electric field, resulting in a current larger than the dark current. Figure 4c exhibits the $I_{\mathrm{ds}}-V_{\mathrm{ds}}$ curves with and without laser illumination $(2.8 \mu \mathrm{m}, 37.1 \mu \mathrm{W} ; 4.2 \mu \mathrm{m}, 40.6 \mu \mathrm{W}$; $10 \mu \mathrm{m}, 41.6 \mu \mathrm{W})$. Different from the photovoltaic effect, a photocurrent is generated at forward bias. In addition, the photocurrent increases with $V_{\mathrm{ds}}$ and shows no indication of saturation, which further confirms the bolometric mechanism. The photocurrent under $10 \mu \mathrm{m}$ laser illumination is larger than that at the other two wavelengths, which can be ascribed to the reflectivity of $\mathrm{VO}_{2}$ at $10 \mu \mathrm{m}$ being smaller than that at 2.8 and $4.2 \mu \mathrm{m}^{49}$. In other words, the device is more suitable for detecting LWIR. The responsivity is calculated at different forward biases, as shown in the inset of Fig. 4c. The responsivity under $2.8 \mu \mathrm{m}$ laser illumination is slightly larger than that under $4.2 \mu \mathrm{m}$ when $V_{\mathrm{ds}}<0.2 \mathrm{~V}$, which we think is due to the electronic transition of impurities and defect bands in the polycrystalline thin film, as confirmed by the absorption spectrum in Fig. S6b.

Figure $4 \mathrm{~d}$ shows the time-resolved response at $V_{\mathrm{ds}}=1 \mathrm{~V}$ under laser illumination of three wavelengths. The currents show an obvious contrast with and without laser illumination, although the rise and fall times are slightly long, which is determined by the heat capacity of the device and substrate. Effective methods can be used to enhance the performance, such as thinning the substrate thickness and introducing a suspended support ${ }^{50}$. The power dependence is investigated by measuring $I_{\mathrm{ds}}-V_{\mathrm{ds}}$ 
curves under different laser powers, as shown in Fig. S9. Figure $\mathrm{S} 9$ also exhibits the $\left|I_{\mathrm{ds}}\right|-V_{\mathrm{ds}}$ curves on a logarithmic ordinate, in which the current at reverse bias is also larger than the dark current. This occurs because the thermal equilibrium is influenced as well as the Fermi level, with the result being an enlarged reverse saturation current under laser illumination. Figure $4 \mathrm{e}$ shows the calculated responsivity at different powers. The responsivity for each wavelength remains almost constant, which indicates the stability of our device. However, the responsivity suddenly increases from 0.06 to $0.47 \mathrm{~A} / \mathrm{W}$ at $157 \mu \mathrm{W}$. This drastic change occurs during the MIT because the absorbed laser energy exceeds the energy for $\mathrm{VO}_{2}$ to undergo a transition from a semiconductor to a metal. The time-resolved photoresponse under $157 \mu \mathrm{W}$ of $4.2 \mu \mathrm{m}$ light is shown in Fig. 4f. There are two stages in the increase in the current, corresponding to the slopes in the inset of Fig. 4a. $I_{\mathrm{ds}}$ increases slowly at first and then increases rapidly when the absorbed energy reaches the energy required for the phase transition. This abrupt increase in current also indicates the transformation of our device from the $\mathrm{p}-\mathrm{n}$ junction to the Schottky junction. Finally, the current tends to saturate because $\mathrm{VO}_{2}$ has completely changed into a metal, and its electric resistance changes little under laser illumination. When the laser is removed, the current rapidly decreases due to the rapid phase transition of $\mathrm{VO}_{2}$. Based on this phenomenon, the device can also be used for the detection of ultrahigh laser power.

\section{Schottky junction mode}

As discussed above, the device transforms to a Schottky junction when the laser power exceeds the MIT energy. Here, the process of the mode changing with temperature and the performance of the Schottky junction mode are comprehensively investigated. Figure 5a shows the $I_{\mathrm{ds}}-$ $V_{\mathrm{ds}}$ curves from 300 to $400 \mathrm{~K}$. The current rapidly increases from 350 to $360 \mathrm{~K}$, indicating the mode switching of the device. The inset shows the calculated ideality factor $n$, which suddenly changes from 2.2 to 2.5 , corresponding to the $\mathrm{p}-\mathrm{n}$ junction transforming to the Schottky junction. In the Schottky junction, electrons diffuse from $\mathrm{MoTe}_{2}$ to the interface when the Fermi levels are equalized. The SCR appears on the side of the semiconductor, forming a built-in electric field directed from $\mathrm{MoTe}_{2}$ to the surface of $\mathrm{VO}_{2}$. Under laser illumination, the electrons of $\mathrm{MoTe}_{2}$ can be excited to the valance band, leaving an electron-hole pair. The electron-hole pair is then separated by the built-in electric field (Fig. 5b), which seems similar to the $\mathrm{p}-\mathrm{n}$ junction at ambient temperature. However, as $\mathrm{VO}_{2}$ has completely transformed into a metal, the cutoff wavelength is determined by the bandgap of $\mathrm{MoTe}_{2}$ rather than $\mathrm{VO}_{2}$. Figure $5 \mathrm{c}$ presents the $\left|I_{\mathrm{ds}}\right|-V_{\mathrm{ds}}$ curves with and without laser illumination $(830 \mathrm{~nm}, 15.7 \mu \mathrm{W})$ at $400 \mathrm{~K}$. The reverse dark current increases with the bias due to the lowering of the barrier, which is the significant difference with the $\mathrm{p}-\mathrm{n}$ junction. Figure $5 \mathrm{~d}$ shows the temporal response under laser illumination in which the dark current increases from $0.2 \mathrm{pA}$ to $0.2 \mathrm{nA}$. The increased dark current seems to degrade the performance of the photodetector, however, the significant on/off ratio as large as $10^{4}$ makes it still able to function as a photodetector. As shown in Fig. $5 \mathrm{e}$, the rise and fall times are measured and evaluated as 45 and $88 \mu \mathrm{s}$, respectively, which are longer than those in the $\mathrm{p}-\mathrm{n}$ junction mode. The performance of the device for different illumination intensities is exhibited in Fig. S10. It can be seen that $I_{\mathrm{sc}}$ significantly increases, especially in logarithmic coordinates, at zero bias. The $V_{\mathrm{oc}}$ values extracted from $I_{\mathrm{ds}}-V_{\mathrm{ds}}$ curves are plotted and fitted as a function of $\log (P)$. The interlayer recombination order $\beta$ is calculated to be 2.57 , indicating a bimolecular recombination process, different from the monomolecular process in the above $\mathrm{p}-\mathrm{n}$ junction. The power dependence and responsivity are further investigated at $400 \mathrm{~K} . I_{\mathrm{ph}}$ scales linearly with power, and no evidence of saturation is observed in the measured range. The responsivity is calculated to be $\sim 0.12 \mathrm{~A} / \mathrm{W}$. The reduced performance results from the photons only being absorbed by $\mathrm{MoTe}_{2}$, while metallic $\mathrm{VO}_{2}$ contributes nothing. Due to the metallization of $\mathrm{VO}_{2}, \mathrm{EQE}$ also decreases to $\sim 18 \%$ (Fig. S10). The relatively outstanding performance under high temperature is definitely a breakthrough in the infrared detector.

\section{Discussion}

A unique heterostructure based on $\mathrm{VO}_{2}$ and $\mathrm{MoTe}_{2}$ is demonstrated. As self-synthesized $\mathrm{VO}_{2}$ behaves as a ptype semiconductor and thin $\mathrm{MoTe}_{2}$ behaves as an n-type material, the heterostructure is a perfect $\mathrm{p}-\mathrm{n}$ junction that acts as an ultrasensitive and ultrafast detector. A forward bias makes it switch to bolometer mode, capable of detecting broadband radiation from MWIR to LWIR. In addition, high temperatures above $T_{c}$ lead to the transformation of $\mathrm{VO}_{2}$ from a semiconductor to a metal, resulting in the third operating mode of the Schottky junction, which is stable under extreme conditions with prominent performance. The heterostructure takes advantage of the photovoltaic effect and bolometric effect, exhibiting multiple functions covering the visible to LWIR, which is a promising approach for advanced uncooled photodetectors.

\section{Materials and methods Materials and characterization}

The $\mathrm{VO}_{2}$ film was synthesized by direct current magnetron sputtering and thermal oxidation. First, a metal vanadium thin film was deposited under an Ar atmosphere 

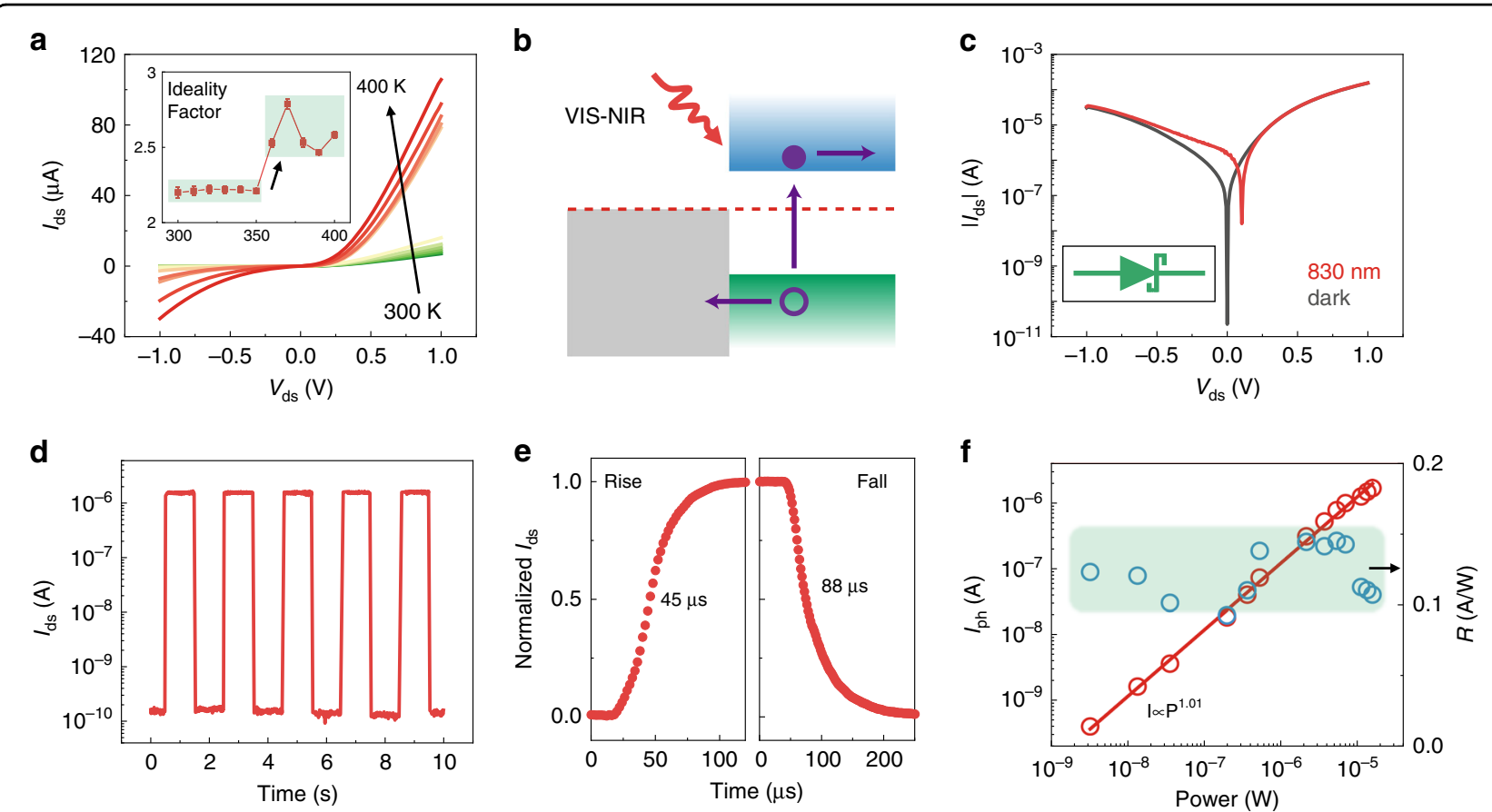

Fig. 5 Schottky junction mode of the heterostructure. $\mathbf{a} I_{d s}-V_{d s}$ curves under temperatures from 300 to $400 \mathrm{~K}$. The inset shows the ideality factor as a function of temperature. $\mathbf{b}$ Schematic of the photovoltaic mechanism of the Schottky junction for detecting VIS to NIR light. $\mathbf{c} I_{d s}-V_{d s}$ curves on a logarithmic scale under the dark state and $830 \mathrm{~nm}$ laser illumination. $\mathbf{d}$ Temporal response of the photocurrent under laser illumination at $830 \mathrm{~nm}$; the power is $15.7 \mu \mathrm{W}$. e Rise and fall times of the normalized photocurrent under zero bias measured by an oscilloscope. $\mathbf{f} /$ ph and responsivity as a function of the incident laser power.

of $1.2 \times 10^{-1} \mathrm{~Pa}$. The obtained metal vanadium film was then put into an annealing furnace for oxidation in a mixture gas of Ar and air $(70 \mathrm{sccm})$ at $470^{\circ} \mathrm{C}$.

$\mathrm{MoTe}_{2}$ was purchased from 2D Semiconductors Inc. and thinned by mechanical exfoliation.

All the morphological structures of the devices were characterized by a Nikon optical microscope. AFM images were taken by a Bruker Dimension Edge in tapping mode. XRD measurements were performed by using a Bruker D8 Discover. The Raman spectra were obtained by a Lab Ram HR800 from HORIBA with a $514 \mathrm{~nm}$ excitation laser. The TEM images and EDS mapping were obtained by a JEOL JEM2100F TEM with an EX-24063JGT EDS.

\section{Device fabrication and measurements}

The heterostructure devices were fabricated by restacking techniques. First, the $\mathrm{VO}_{2}$ film was patterned by UV photolithography and Ar plasma etching. $\mathrm{MoTe}_{2}$ flakes were exfoliated by scotch tape onto polydimethylsiloxane and transferred to the top of etched $\mathrm{VO}_{2}$ by dry transfer ${ }^{12}$. Polymethyl methacrylate and conductive ink were spin-coated ( $4000 \mathrm{rpm}$ for $45 \mathrm{~s}$ ) on the substrate for electron beam lithography to define electrodes. $\mathrm{Pt} / \mathrm{Au}$ $(20 / 60 \mathrm{~nm})$ was sputtered as electrodes on $\mathrm{MoTe}_{2}$ and $\mathrm{VO}_{2}$ to form ohmic contacts. After the lift-off process, a heterostructure device was obtained.
The electronic and temperature dependence measurements of $\mathrm{VO}_{2}$ were performed by using an Agilent B2902A in a vacuum chamber probe station with a temperature control unit (K2000, MMR Technologies, Inc.). The electronic and optoelectronic measurements from $450 \mathrm{~nm}$ to $2 \mu \mathrm{m}$ were conducted using a Keysight B1500A semiconductor parameter analyzer on a Lake Shore probe station. The response time data were acquired from a high-speed Tektronix (Tektronix MDO3014). The optoelectronic measurements at $2.8,4.2$, and $10 \mu \mathrm{m}$ and scanning photocurrent microscopy were performed with a homemade optoelectronic measurement system.

\section{Acknowledgements}

This work is supported by the Natural Science Foundation of China (Grant Nos. 61835012, 61722408, 61725505, 61521005, and 61905267), the Key Research Project of Frontier Sciences of the Chinese Academy of Sciences (Grant Nos. QYZDB-SSW-JSC016 and QYZDY-SSW-JSC042), the Key Research Program of Frontier Science, CAS (Grant No. ZDBS-LY-JSC045), the Major State Basic Research Development Program (Grant No. 2016YFA0203900), the National Postdoctoral Program for Innovative Talents (BX20180329) and the Natural Science Foundation of Shanghai (Grant Nos. $16 Z$ R1447600 and 17JC1400302), the Strategic Priority Research Program of the Chinese Academy of Sciences (Grant No. XDB44000000).

\section{Author details}

'State Key Laboratory of Infrared Physics, Shanghai Institute of Technical Physics, Chinese Academy of Sciences, 500 Yutian Road, Shanghai 200083, China. ${ }^{2}$ University of Chinese Academy of Sciences, Beijing 100049, China. 
${ }^{3}$ Department of Applied Physics, Donghua University, No. 2999, North Renmin Road, Songjiang District, Shanghai 201620, China

\section{Author contributions}

J.W. conceived the concept and designed the experiments. W.J. performed device fabrication and characterization. T.Z. and X.X. synthesized $\mathrm{VO}_{2}$ film. B.W., X.J., X.W., and M.P. helped with measurement of devices. Y.C. helped with the restacking technique of device fabrication. X.Z. and H.W. performed the XRD and AFM characterization. All authors discussed the results and revised the manuscript.

\section{Conflict of interest}

The authors declare that they have no conflict of interest.

Supplementary information is available for this paper at https://doi.org/ 10.1038/s41377-020-00396-3.

Received: 6 May 2020 Revised: 31 July 2020 Accepted: 24 August 2020 Published online: 10 September 2020

\section{References}

1. Rogalski A. Infrared detectors: status and trends. Prog. Quantum Electron. 27, 59-210 (2003).

2. Rogalski A. Next decade in infrared detectors. In Proc. SPIE, Electro-Optical and Infrared Systems: Technology and Applications XIV (SPIE, Warsaw, 2017).

3. Rogalski A. Infrared detectors for the future. Acta Phys. Polonica A 116 389-406 (2009).

4. Rogalski, A. \& Chrzanowski, K. Infrared devices and techniques. Opto-Electron. Rev. 10, 111-136 (2002)

5. Rogalski A. Semiconductor detectors and focal plane arrays for far-infrared imaging. Opto-Electron. Rev. 21, 406-426 (2013).

6. Rogalski A. HgCdTe infrared detector material: history, status and outlook. Rep. Prog. Phys. 68, 2267-2336 (2005).

7. McDonald S. A. et al. Solution-processed PbS quantum dot infrared photodetectors and photovoltaics. Nat. Mater. 4, 138-142 (2005).

8. Miao J. S. et al. Single InAs nanowire room-temperature near-infrared photodetectors. ACS Nano 8, 3628-3635 (2014).

9. Liu C. H. et al. Graphene photodetectors with ultra-broadband and high responsivity at room temperature. Nat. Nanotechnol. 9, 273-278 (2014).

10. Arora K. et al. Ultrahigh performance of self-powered $\beta-\mathrm{Ga}_{2} \mathrm{O}_{3}$ thin film solarblind photodetector grown on cost-effective $\mathrm{Si}$ substrate using hightemperature seed layer. ACS Photonics 5, 2391-2401 (2018).

11. Kumar, N., Arora, K. \& Kumar, M. High performance, flexible and room temperature grown amorphous $\mathrm{Ga}_{2} \mathrm{O}_{3}$ solar-blind photodetector with amorphous indium-zinc-oxide transparent conducting Electrodes. J. Phys. D: Appl. Phys. 52, 335103 (2019)

12. Arora K. et al. Spectrally selective and highly sensitive UV photodetection with UV-A, C band specific polarity switching in silver plasmonic nanoparticle enhanced gallium oxide thin-film. Adv. Opt. Mater. https://doi.org/10.1002/ adom.202000212 (2020).

13. Geim, A. K. \& Grigorieva, I. V. Van der Waals heterostructures. Nature 499, 419-425 (2013).

14. Liu Y. et al. Van der Waals heterostructures and devices. Nat. Rev. Mater. 1 16042 (2016).

15. Goossens S. et al. Broadband image sensor array based on graphene-CMOS integration. Nat. Photonics 11, 366-371 (2017).

16. Wang Q. H. et al. Electronics and optoelectronics of two-dimensional transition metal dichalcogenides. Nat. Nanotechnol. 7, 699-712 (2012).

17. Koppens F. H. L. et al. Photodetectors based on graphene, other twodimensional materials and hybrid systems. Nat. Nanotechnol. 9, 780-793 (2014).

18. Xia F. N. et al. Ultrafast graphene photodetector. Nat. Nanotechnol. 4, 839-843 (2009).

19. Lopez-Sanchez O. et al. Ultrasensitive photodetectors based on monolayer $\mathrm{MoS}_{2}$. Nat. Nanotechnol. 8, 497-501 (2013).

20. Yuan H. T. et al. Polarization-sensitive broadband photodetector using a black phosphorus vertical p-n junction. Nat. Nanotechnol. 10, 707-713 (2015).
21. Wang J. L. et al. Recent progress on localized field enhanced two-dimensional material photodetectors from ultraviolet-visible to infrared. Small 13, 1700894 (2017).

22. Miao J. S. et al. Gate-tunable Semiconductor heterojunctions from 2D/3D van der Waals interfaces. Nano Lett. 20, 2907-2915 (2020).

23. Rao G. F. et al. Two-dimensional heterostructure promoted infrared photodetection devices. InfoMat 1, 272-288 (2019).

24. Jariwala, D., Marks, T. J. \& Hersam, M. C. Mixed-dimensional van der Waals heterostructures. Nat. Mater. 16, 170-181 (2017).

25. Morin F. J. Oxides which show a metal-to-insulator transition at the neel temperature. Phys. Rev. Lett. 3, 34-36 (1959).

26. Shin S. et al. Vacuum-ultraviolet reflectance and photoemission study of the metal-insulator phase transitions in $\mathrm{VO}_{2}, \mathrm{~V}_{6} \mathrm{O}_{13}$, and $\mathrm{V}_{2} \mathrm{O}_{3}$. Phys. Rev. B 41, 4993-5009 (1990)

27. Qazilbash M. M. et al. Mott transition in $\mathrm{VO}_{2}$ revealed by infrared spectroscopy and nano-Imaging. Science 318, 1750-1753 (2007).

28. Heckingbottom, R. \& Linnett, J. W. Structure of vanadium dioxide. Nature 194, 678 (1962)

29. Aetukuri N. B. et al. Control of the metal-insulator transition in vanadium dioxide by modifying orbital occupancy. Nat. Phys. 9, 661-666 (2013).

30. Dou Y. K. et al. Oxidizing annealing effects on $\mathrm{VO}_{2}$ films with different microstructures. Appl. Surf. Sci. 345, 232-237 (2015).

31. Petrov, G. I., Yakovlev, V. V. \& Squier, J. Raman microscopy analysis of phase transformation mechanisms in vanadium dioxide. Appl. Phys. Lett. 81, 1023-1025 (2002).

32. Cao J. et al. Strain engineering and one-dimensional organization of metalinsulator domains in single-crystal vanadium dioxide beams. Nat. Nanotechnol. 4, 732-737 (2009).

33. Wei J. et al. New aspects of the metal-insulator transition in single-domain vanadium dioxide nanobeams. Nat. Nanotechnol. 4, 420-424 (2009).

34. Chen C. H. et al. Influence of defects on structural and electrical properties of $\mathrm{VO}_{2}$ thin films. J. Appl. Phys. 110, 023707 (2011).

35. Mleczko M. J. et al. Contact engineering high-performance n-type MoTe transistors. Nano Lett. 19, 6352-6362 (2019).

36. Froehlicher $\mathrm{G}$. et al. Unified description of the optical phonon modes in $\mathrm{N}$ layer MoTe. Nano Lett. 15, 6481-6489 (2015).

37. Puotinen, D. \& Newnham, R. E. The crystal structure of MoTe2. Acta Crystallogr. 14, 691-692 (1961)

38. Longo, J. M. \& Kierkegaard, P. A refinement of the structure of $\mathrm{VO}_{2}$. Acta Chem Scandinavica. 24, 420-426 (1970).

39. Baugher B. W. H. et al. Optoelectronic devices based on electrically tunable p-n diodes in a monolayer dichalcogenide. Nat. Nanotechnol. 9, 262-267 (2014).

40. Pospischil, A., Furchi, M. M. \& Mueller, T. Solar-energy conversion and light emission in an atomic monolayer p-n diode. Nat. Nanotechnol. 9, 257-261 (2014).

41. Wang X. D. et al. Ultrasensitive and broadband $\mathrm{MoS}_{2}$ photodetector driven by ferroelectrics. Adv. Mater. 27, 6575-6581 (2015).

42. Furchi M. M. et al. Photovoltaic effect in an electrically tunable van der Waals heterojunction. Nano Lett. 14, 4785-4791 (2014).

43. Chen Y. et al. High-performance photovoltaic detector based on MoTe$/ \mathrm{MoS}_{2}$ van der Waals heterostructure. Small 14, 1703293 (2018)

44. Jin Y. et al. A van der Waals homojunction: ideal p-n diode behavior in MoSe Adv. Mater. 27, 5534-5540 (2015).

45. Zhang K. A. et al. Interlayer transition and infrared photodetection in atomically thin type-II MoTe$/ \mathrm{MoS}_{2}$ van der Waals Heterostructures. ACS Nano 10, 3852-3858 (2016).

46. Wu D. et al. Design of $2 \mathrm{D}$ layered $\mathrm{PtSe}_{2}$ heterojunction for the high-performance, room-temperature, broadband, infrared photodetector. ACS Photonics 5, 3820-3827 (2018).

47. Li Z. J. et al. Ultrahigh infrared photoresponse from core-shell single-domain$\mathrm{VO}_{2} \mathrm{~N}_{2} \mathrm{O}_{5}$ heterostructure in nanobeam. Adv. Funct. Mater. 24, 1821-1830 (2014).

48. Jariwala D. et al. Gate-tunable carbon nanotube-MoS, heterojunction p-n diode. Proc. Natl Acad. Sci. U. S. A. 110, 18076-18080 (2013).

49. Barker, A. S., Verleur, H. W. Jr \& Guggenheim, H. J. Infrared optical properties of vanadium dioxide above and below the transition temperature. Phys. Rev. Lett. 17, 1286-1289 (1966)

50. Niklaus F. MEMS-based uncooled infrared bolometer arrays-a review. In Proc SPIE, MEMS/MOEMS Technologies and Applications III (SPIE, Beijing, 2007). 